\title{
Price-based pilot sharing for spectrum sharing massive MIMO networks
}

\author{
Wanming Hao ${ }^{1 \mathrm{a})}$, Osamu Muta ${ }^{1}$, Haris Gacanin ${ }^{2}$, \\ and Hiroshi Furukawa ${ }^{1}$ \\ ${ }^{1}$ Kyushu University, Fukuoka-shi, Fukuoka, Japan \\ ${ }^{2}$ Nokia Bell Labs, Belgium \\ a)hao@mobcom.ait.kyushu-u.ac.jp
}

Abstract: This letter studies a pilot sharing scheme for spectrum-sharing massive MIMO networks, where primary network (PN) can lease portion of orthogonal pilots to secondary network (SN) for channel estimation. We assume that $\mathrm{PN}$ and $\mathrm{SN}$ are rational and selfish, and they aim at maximizing their revenue when pilots are traded. Then, we propose a price-based iterative optimal pilot allocation algorithm to obtain win-win paradigm, while guaranteeing the primary user ratio (PUR) of the primary cell. Simulation results reveal that PN can achieve more revenue by sacrificing limited pilots while decreasing total interference to adjacent cells.

Keywords: pilot allocation, primary network, secondary network, massive MIMO, lease price

Classification: Wireless Communication Technologies

\section{References}

[1] L. Lu, G. Y. Li, A. L. Swindlehurst, A. Ashikhmin, and R. Zhang, "An overview of massive MIMO: Benefits and challenges," IEEE J. Sel. Areas Commun., vol. 8, no. 5, pp. 742-758, Oct. 2014. DOI:10.1109/JSTSP.2014.2317671

[2] S. Haykin, "Cognitive radio: Brain-empowered wireless communications," IEEE J. Sel. Areas Commun., vol. 23, no. 2, pp. 201-220, Feb. 2005. DOI:10. 1109/JSAC.2004.839380

[3] L. Sboui, Z. Rezki, and M. S. Alouini, "A unified framework for the ergodic capacity of spectrum sharing cognitive radio systems," IEEE Trans. Wireless Commun., vol. 12, no. 2, pp. 877-887, Feb. 2013. DOI:10.1109/TWC.2012. 122212.120449

[4] M. Filippou, D. Gesbert, and H. Yin, "Decontaminating pilots in cognitive massive MIMO networks," Int. Symp. Wireless Commun. Syst. Conf., France, Pairs, pp. 816-820, Aug. 2012. DOI:10.1109/ISWCS.2012.6328481

[5] B. Kouassi, I. Ghauri, and L. Deneire, "Reciprocity-based cognitive transmissions using a MU massive MIMO approach," IEEE Int. Conf. Commun. (ICC), Hungary, Budapest, pp. 2738-2742, May 2013. DOI:10.1109/ICC.2013. 6654952 


\section{Introduction}

Massive multiple-input-multiple-output (mMIMO) has been a candidate technology for future wireless communication systems [1], where each base-station (BS) is equipped with a large number of antennas and serves users with the same timefrequency resource. As another approach, cognitive radio (CR) has attracted much research interest for its ability to improve spectrum utilization [2]. However, current studies mainly focus on traditional MIMO-CR system with regular antennas. In [3], the authors considered the achievable rate and power efficiency for mMIMO in spectrum-sharing networks. For maximizing the quality of channel estimation for secondary-network (SN), a pilot decontamination algorithm was proposed [4]. In [5], a reciprocity-based CR beamforming scheme was proposed to reduce the interference from secondary-users (SUs) to primary-users (PUs). However, works in $[3,4,5]$ does not involve the pilot allocation problem between primary-network $(\mathrm{PN})$ and $\mathrm{SN}$.

Unlike previous works, we study pilot sharing problem in spectrum-sharing mMIMO networks. In our approach, PN and SN are regarded as the leaser and lessee, respectively. The SN is allowed to lease a part of available orthogonal pilots from PN. Consequently, PN can obtain profits by leasing pilots to SN. We assume that $\mathrm{PN}$ and $\mathrm{SN}$ are rational and selfish, and they aim at maximizing their revenue when pilots are traded. To guarantee success of pilot trade, we propose a three-side pilot trade platform, including price control side (PCS), PN side and SN side. Specifically, for given pilot lease price, PN side will lease the optimum pilots to SN for maximizing its revenue. Then, SN side allocates these pilots to some of SUs for maximizing its revenue. To realize the above, we propose a price-based iterative optimum pilot allocation algorithm to maximize the profits of PN and SN.

\section{System model and proposed scheme}

We consider a communication system that consists of one $L$ hexagonal primarycells (PCs) PN and one single hexagonal secondary-cell (SC) SN, as shown in Fig. 1. We assume that $\mathrm{SC}$ has the same coverage area with the central PC. For convenience, the central PC and SC are denoted as the 1st PC and SC (target cell), respectively. Each PC consists of a primary BS (PBS) equipped with $M$ antennas and $K(\mathcal{K}=\{1, \ldots, K\})$ single-antenna PUs $(M \gg K)$. We assume that the same $K$ orthogonal pilots sequences $\boldsymbol{\Psi}=\left\{\psi_{1}, \ldots, \boldsymbol{\psi}_{K}\right\} \in \mathbb{C}^{K \times K}$ are assigned to $K$ PUs uniquely in each PC, where $\boldsymbol{\Psi} \boldsymbol{\Psi}^{H}=\mathbf{I}_{M}$, and no pilots are assigned to SUs. $\psi_{\theta(l, k)}(\theta(l, k) \in\{1, \ldots, K\})$ denotes that the $\theta(l, k)$-th pilot is used by the $k$-th PU in the $l$-th PC, where $\theta(l, k) \neq \theta\left(l, k^{\prime}\right)$ when $k \neq k^{\prime}$. Uplink rate of the $k$-th PU in the 1 st PC can be expressed as [1]

$$
r_{1, k}^{p}=\log _{2}\left(1+\operatorname{SINR}_{1, k}^{p}\right),
$$

where $\operatorname{SINR}_{1, k}^{p} \approx \frac{\beta_{11 k}^{p}{ }^{2}}{\sum_{l \neq 1}^{L} \sum_{i=1}^{K} f(\theta(l, i), \theta(1, k)) \beta_{1 l i}^{p}{ }^{2}}$ when $M \rightarrow \infty, \beta_{1 l i}^{p}$ denotes the largescale fading coefficient (LFC) between the PBS in the 1st PC and the $i$-th PU in the $l$-th PC. $\beta_{1 l i}^{p}=1 / d_{1 l i}^{p \alpha}$, where $d_{1 l i}^{p}$ denotes the distance between PBS in the 1 st $\mathrm{PC}$ and the $i$-th $\mathrm{PU}$ in the $l$-th $\mathrm{PC}$, and $\alpha$ is path-loss exponent. 


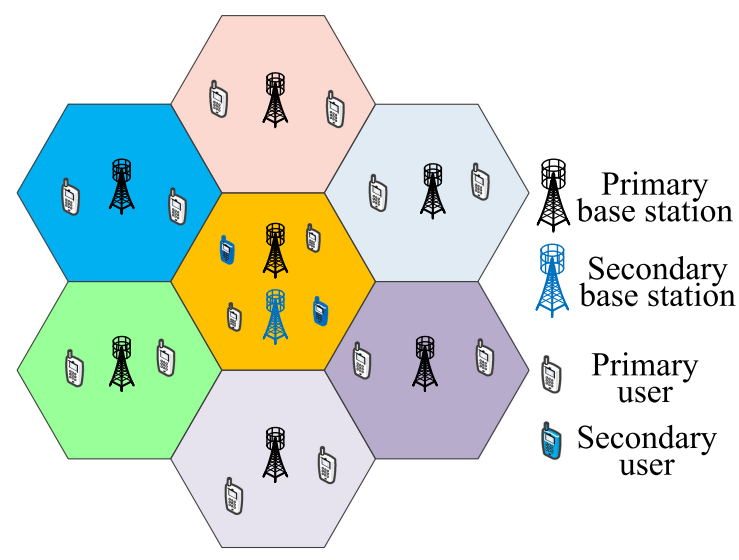

Fig. 1. System model for spectrum-sharing mMIMO networks.

$\sum_{j \neq l} \sum_{i=1}^{K} f(\theta(j, i), \theta(1, k)) \beta_{1 j i}^{p}{ }^{2}$ denotes pilot contamination caused by pilot reuse in adjacent cells, $f(\theta(j, i), \theta(l, k))=1$ when $\theta(j, i)=\theta(l, k)$, else $f(\theta(j, i)$, $\theta(l, k))=0$.

In $\mathrm{SC}$, there are a $M$-antenna secondary BS (SBS) and $K$ single-antenna SUs. Similarly, uplink rate of the $k$-th $\mathrm{SU}$ in the $\mathrm{SC}$ can be expressed as $r_{1, k}^{s}=$ $\log _{2}\left(1+\operatorname{SINR}_{1, k}^{s}\right)$, where $\operatorname{SINR}_{1, k}^{s} \approx \frac{\beta_{11 k}^{s}{ }^{2}}{\sum_{l \neq 1}^{L} \sum_{i=1}^{K} f(\theta(l, i), \theta(1, k)) \beta_{1 l i}^{s}{ }^{2}}$ when $M \rightarrow \infty, \beta_{11 i}^{s}$ denotes the LFC between SBS and the $i$-th $\mathrm{SU}$ in the $\mathrm{SC}$, and $\beta_{1 l i}^{s}$ denotes the LFC between SBS in the 1 st PC and the $i$-th PU in the $l$-th PC.

$\mathrm{SN}$ can lease pilots from $\mathrm{PN}$ for channel estimation. PN can get some revenue from SN (i.e., lease fee). We assume that PN and SN are rational and selfish. For given pilot lease price, $\mathrm{PN}$ always leases optimum pilots to $\mathrm{SN}$ for maximizing its revenue, while SN always optimally allocates these pilots to SUs for maximizing its own revenue.

Since we assume that SC has the same coverage area with the 1st PC, SN leases pilots from the 1st PC. Therefore, the revenue of PN can be given as follows:

$$
\begin{gathered}
\max _{\boldsymbol{\Psi}_{\mathrm{S}}, \mathcal{K}_{\mathrm{P}}} \underbrace{m\left|\boldsymbol{\Psi}_{\mathrm{S}}\right|}_{\begin{array}{c}
\text { Price of leased pilots } \\
\text { from PN to SN }
\end{array}}-n \underbrace{\sum_{i \in \mathcal{K}_{\mathrm{P}}, \psi_{\theta(1, i)} \in \boldsymbol{\Psi}_{\mathrm{S}}} r_{1, i}^{p},}_{\text {Lost utility (PUs' rate) }} \\
=\max _{\boldsymbol{\Psi}_{\mathrm{S}}, m} m\left|\boldsymbol{\Psi}_{\mathrm{S}}\right|-\min _{\mathbf{\Psi}_{\mathrm{S}}, \mathcal{K}_{\mathrm{P}}} n \sum_{\substack{i \in \mathcal{K}_{\mathrm{P}} \\
\psi_{\theta(1, i)} \in \boldsymbol{\Psi}_{\mathrm{S}}}} r_{1, i}^{p},
\end{gathered}
$$

where $m$ is the lease price per pilot, $n$ denotes the price per rate of PN which is determined by PN. $\boldsymbol{\Psi}_{\mathrm{S}}\left(\boldsymbol{\Psi}_{\mathrm{S}} \subset \boldsymbol{\Psi}\right)$ and $\left|\boldsymbol{\Psi}_{\mathrm{S}}\right|$ denote the leased pilots set to SN and the number in set $\boldsymbol{\Psi}_{\mathrm{S}}$, respectively. $\mathcal{K}_{\mathrm{P}}\left(\mathcal{K}_{\mathrm{P}} \subset \mathcal{K}\right)$ denotes the pilots allocated to PUs in the 1 st PC before pilots leasing. In $(2 \mathrm{a})$, the first term denotes the obtained revenue because of leased pilots. The second term denotes the lost utility because those PUs will not connect the PBS. From (2b), we can find that the first term is a increasing linear function with $\left|\Psi_{\mathrm{S}}\right|$ for given $m$, and the second term is a function of growing faster with $\left|\boldsymbol{\Psi}_{\mathrm{S}}\right|$. Therefore, for given pilot lease price, the 1 st PC can find the optimum pilots to the SN for maximizing its revenue. Since there is no relationship between the secondary term and $m,(2 \mathrm{~b})$ is a monotone non-decreasing function with $m$. This analysis assumes that users are randomly distributed in each cell. 
At the SN side, for given pilot price $m$ and pilots $\boldsymbol{\Psi}_{\mathrm{S}}$, the total revenue can be expressed as:

$$
\begin{aligned}
& \max _{\mathcal{K}_{\mathrm{S}}} c \underbrace{c \sum_{\substack{i \in \mathcal{K}_{\mathrm{S}} \\
\psi_{\theta(1, i} \in \mathbf{\Psi}_{\mathrm{S}}}} r_{1, i}^{\mathrm{s}}}_{\text {Obtained utility (SUs' rate) }}-\underbrace{m\left|\boldsymbol{\Psi}_{\mathrm{S}}\right|}_{\begin{array}{c}
\text { Fefor leased pilots } \\
\text { from PN to SN }
\end{array}}, \\
& \triangleq \max _{\mathcal{K}_{\mathrm{S}}} c \sum_{\substack{i \in \mathcal{K}_{\mathrm{S}} \\
\psi(1, i) \in}} \frac{\beta_{\mathbf{\Psi}_{\mathrm{S}}}^{s}{ }^{2}{ }^{2}}{\sum_{j \neq 1} \sum_{m=1}^{K} f(\theta(j, m), \theta(1, i)) \beta_{1 j m}^{s}{ }^{2}},
\end{aligned}
$$

where $c$ denotes the price per rate of $\mathrm{SN}$ which is determined by $\mathrm{SN}$, and $\mathcal{K}_{\mathrm{S}}\left(\mathcal{K}_{\mathrm{S}} \subset \mathcal{K}\right)$ is a set of SUs allocated pilots. In (3a), the first term denotes obtained utility because of leased pilots from the 1st PC. To obtain more revenue, SN will optimally allocate these pilots to some of SUs. Since the second term in (3a) is given constant, we only need to maximize (3b). (3b) is an optimal matching problem, and matching rule is that one pilot is assigned to one SU, but different users are not assigned the same pilot. To maximize SUs' total SINR, (3b) can be solved by applying the well-known Hungarian algorithm.

To guarantee the 1st PC's service quality, number of available pilots in SN must be limited. We define provided primary user ratio (PUR) as number of PUs under service normalized by the number of all PUs in the 1st PC. We also define the required minimum PUR as $p$, which is expressed as:

$$
\left|\Psi_{\mathrm{S}}\right| \leq(1-p) K
$$

where $(1-p) K$ denotes the number of maximum orthogonal pilots leased to the SN.

To ensure the success of pilot trade, we propose a three-side pilot trade platform, including PCS, PN side and SN side, where PCS is in charge of the pilot trade between PN and SN, while PN selects the set $\boldsymbol{\Psi}_{\mathrm{S}}$ only according to $m$ given by PCS. The mechanism to encourage the pilot trade is explained below. According to (2) and (3), if $m$ is decreased, SN's revenue tends to be decreased, while PN's revenue tends to be increased. Consequently, the pilot trade from PN to $\mathrm{SN}$ is encouraged by decreasing $m$. PN and SN are able to know their own users' LFC, respectively. Here, the revenue of SN can not be negative, and the pilot trade will be finished when one of the following conditions is satisfied: $i$ ) (4) becomes tight constraint, $i$ ) revenue of SN becomes 0 . The flow of the proposed algorithm is illustrated in Fig. 2.

\section{Numerical results}

We consider a typical hexagonal cellular network with 7 PCs and 1 SC (as shown in Fig. 1), and each BS is equipped with $M$ antennas ( $M$ goes infinite) [4]. We assume there are 100 PUs in each PC and 100 SUs in SC, and all users are randomly distributed in each cell. The number of orthogonal pilots is 100 . The cell radius is $r_{c}=500$ meters, and the cell-hole radius is $r_{h}=100$ meters (the terminals are not located in this area) [4]. $n=1, c=1$ and $\alpha=3.8$ are assumed. $m, n, c$ and revenue are regarded as the actual currency. 


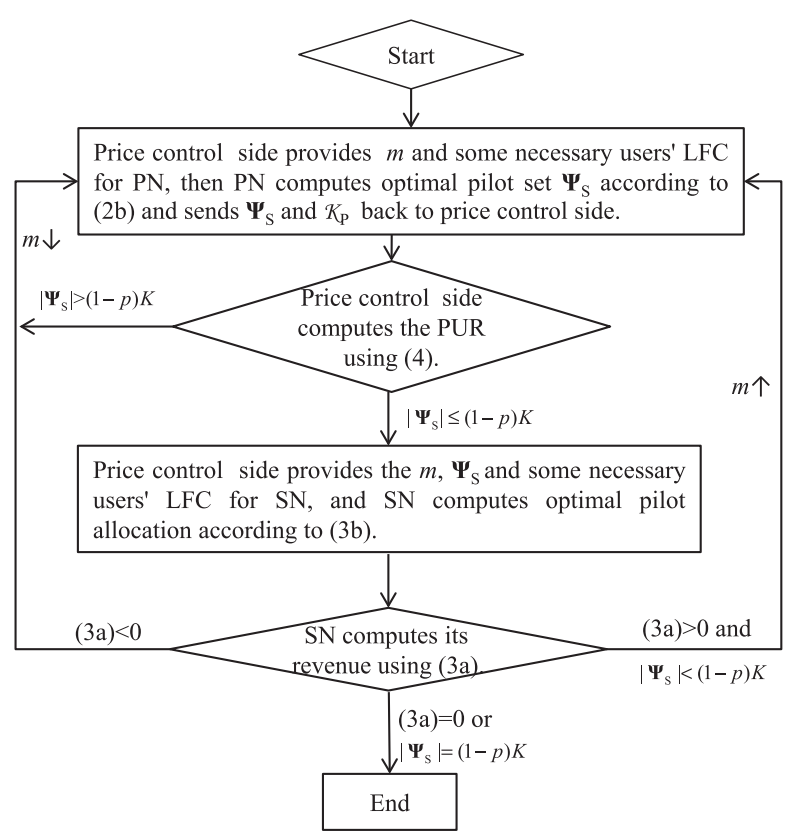

Fig. 2. Proposed pilot allocation algorithm flow diagram.

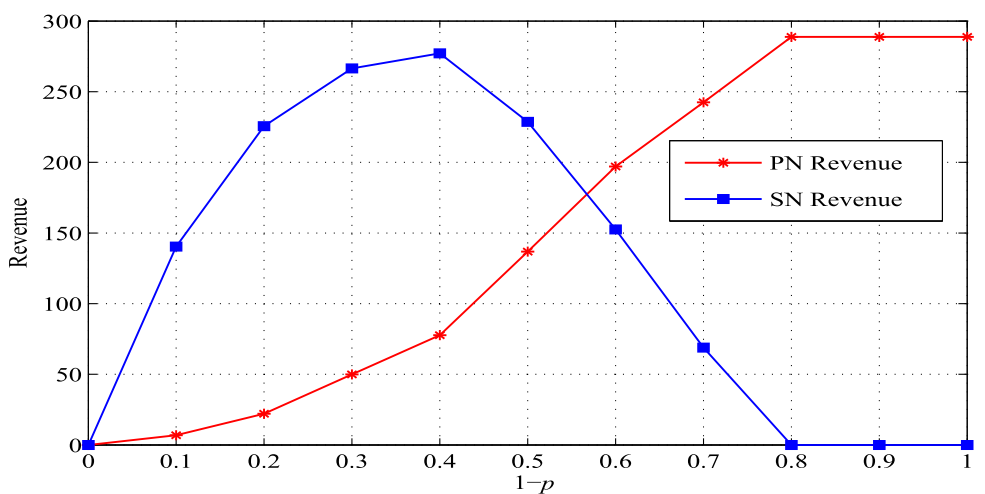

(a) Revenue versus (1-p).

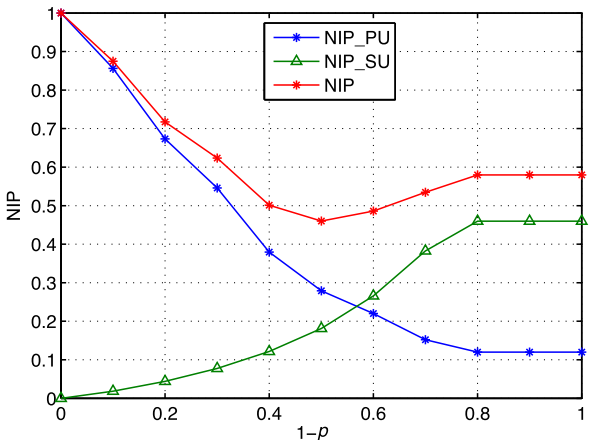

(b) NIP versus $(1-p)$.

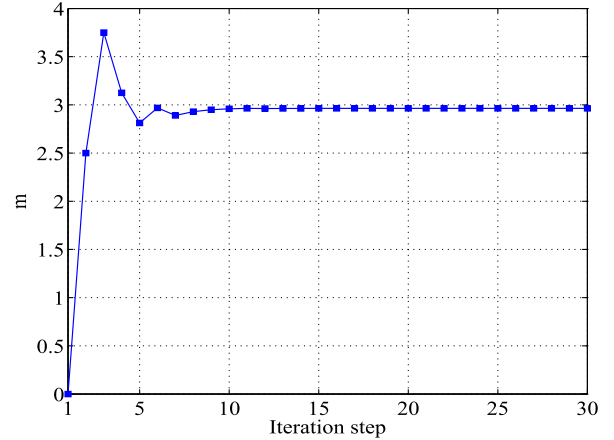

(c) Pilot lease price versus iteration step when $p=0.8$.

Fig. 3. Revenue and NIP curves for $(1-p)$ and the pilot lease price for iteration step.

Fig. 3(a) shows the revenue of PN and SN. We can find that PN's revenue increases with $(1-p)$ and gets maximum at higher $(1-p)$ region. On the other hand, SN's revenue first increases and then decreases with $(1-p)$. This is because 
the first term in (3a) is a logarithmic function with respect to (w.r.t.) $(1-p)$, and the second term in (3a) is a linear function w.r.t. $(1-p)$. Therefore, as $(1-p)$ increases, according to the basic math theory, SN's revenue first increases and then decreases. In Fig. 3(b), we define the normalized interference power (NIP) as $\mathrm{I}_{\text {after }} / \mathrm{I}_{\text {before }}$ :

$$
\mathrm{NIP}=\frac{I_{\text {after }}}{I_{\text {before }}}=\frac{I_{\text {after,PU }}+I_{\text {after,SU }}}{I_{\text {before }}}=\underbrace{\frac{I_{\text {after,PU }}}{I_{\text {before }}}}_{\text {NIP_PU }}+\underbrace{\frac{I_{\text {after,SU }}}{I_{\text {before }}}}_{\text {NIP_SU }},
$$

where $\mathrm{I}_{\text {after }}$ and $\mathrm{I}_{\text {before }}$ denote the total interference power from both PUs and SUs to adjacent PBSs after pilot leasing and before leasing, respectively. $I_{\text {after,PU }}$ and

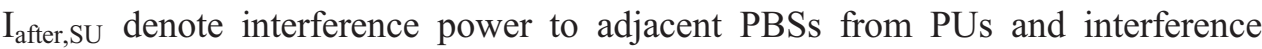
power to adjacent PBSs from SUs, respectively. As the number of leased pilots to SN increases, NIP_PU decreases while NIP_SU increases. Thus, NIP first decreases and then increases, and an optimum value of NIP can be observed for a given $(1-p)=0.5$. As $(1-p)$ increases, more pilots will be leased to SN and less PUs can connect to PBS, which results in the decrease of NIP_PU. On the contrary, as $(1-p)$ increases, more SUs will be allocated pilots and connected to SBS, which leads to the increase of NIP_SU. Note that, NIP_PU, NIP_SU and NIP are kept constant when $(1-p)>0.8$. This is because there is no pilot trade for $(1-p)>$ 0.8 . In addition, the total interference from central cell to adjacent cells is lower compared after pilots leasing. This is because PN will lease those pilots having serious pilot interference to $\mathrm{SN}$, and $\mathrm{SN}$ will allocate those pilots to SUs having the smallest pilot interference, which results in the decrease of total interference after pilot trade. Fig. 3(c) presents the pilot lease price convergence behavior at each iteration when $p=0.8$.

\section{Conclusion}

We have proposed a pilot sharing scheme to achieve a win-win situation for spectrum-sharing mMIMO systems. Simulation results clarified that PN can achieve more revenue by sacrificing limited pilots while decreasing total interference to adjacent cells. 